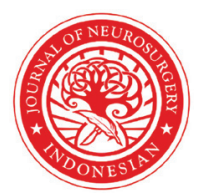

Indonesian Journal of Neurosurgery
${ }^{1}$ Nerosurgery Department, Faculty of Medicine Universitas Indonesia, dr. Cipto Mangunkusumo National Hospital, Jakarta, Indonesia

\section{Mechanical Thrombectomy for Acute Ischemic Stroke in Nonagenarian Patients: A Case Report from dr. Cipto Mangunkusumo National Hospital, Jakarta, Indonesia}

\author{
Affan Priyambodo Permana ${ }^{1 *}$, Zharifah Fauziyyah Nafisah ${ }^{1}$
}

\section{ABSTRACT}

Background: Mechanical thrombectomy is the recommended therapy for acute ischemic stroke (AIS) caused by large vessels occlusion within 6-24 hours since onset. However, previous trials about endovascular treatment for AIS only separated the population into $<80$ years-old and $\geq 80$ years-old groups. Patients greater than 80 years old were found to be associated with poorer clinical outcome and increased mortality. However, in this study we reported a case of revascularization in nonagenarian ( $>90$ years old) with acute ischemic stroke.

Case Presentation: A 93-years-old man with chief complaint of sudden weakness of his left side extremities, slurred speech, and facial palsy since 3.5 hours before admission. In the ER, GCS was E4M6V5 with paresis of $7^{\text {th }}$ and $12^{\text {th }}$ left cranial nerve, and left lateralization of motoric strength. NIHSS score was 12 and code stroke was activated. CT scan showed large vessel occlusion in the right MCA. IV thrombolysis and mechanical thrombectomy was performed using stent retriever. Three clots were successfully removed and $\mathrm{TICI} 3$ was achieved.

Conclusion: Successful reperfusion in elderly and nonagenarian patients was ranging from 56-78\% in several studies. Lower NIHSS at baseline, shorter delay between onset and reperfusion, high ASPECT score on initial brain imaging, and first-pass effect are good predictors of mechanical thrombectomy. Several complications including ICH could happen in $28 \%$ of cases but only $4-7 \%$ patients have symptoms.

Keywords: Mechanical Thrombectomy, Acute Ischemic Stroke, Large Vessel Occlusion, Nonagenarian Cite This Article: Permana, A.P., Nafisah, Z.F. 2021. Mechanical Thrombectomy for Acute Ischemic Stroke in Nonagenarian Patients: A Case Report from dr. Cipto Mangunkusumo National Hospital, Jakarta, Indonesia. Indonesian Journal of Neurosurgery 4(1): 40-42. DOl: 10.15562/ijn.v4i1.148

\section{INTRODUCTION}

Stroke is the leading cause of death in Indonesia due to $\mathrm{WHO}$ data in 2012. ${ }^{1}$ Stroke also the greatest cause of long-term disability in South East Asia. ${ }^{2}$ Ischemic stroke count for $80 \%$ of all stroke cases. Current guideline in management of acute ischemic stroke (AIS) is using IV-thrombolysis (alteplase) as the gold standard therapy for AIS with onset less than 4.5 hours. According to DAWN and DEFUSE trials, large vessel occlusion (LVO) in AIS with onset between 6-24 hours can be treated with mechanical thrombectomy. ${ }^{3}$ Multiple trials already show the benefit of endovascular treatment for acute ischemic stroke, but many studies exclude elderly population ( $>80$ years old), so that mechanical thrombectomy for elderly people, especially nonagenarian is still controversial. ${ }^{4}$ It is expected in the European Union that by 2050 the percentage of elderly population age over 80 years old will be double and the risk of stroke is increasing with age. Age greater than 80 years old was found to be associated with a poorer clinical outcome and increased mortality even the success rate in several studies were comparable to those of younger patients. The oldest documented patient who underwent mechanical thrombectomy for AIS is a 103-years-old woman who recovered weill. ${ }^{5}$ In this study, we report a revascularization in nonagenarian (93-years-old) patient with acute ischemic stroke.

\section{CASE PRESENTATION}

A 93-years-old man came to the emergency department of dr. Cipto Mangunkuusmo National Hospital with chief complaint of sudden weakness of his left side extremities, slurred speech, and facial palsy since 3.5 hours before admission. Patient had no complaint of headache, nausea, vomit, or seizure. In the ER, Glasgow Comma Scale (GCS) was E4M6V5 with paresis of $7^{\text {th }}$ and $12^{\text {th }}$ central left cranial nerve, and hemiplegia of the left extremities. Blood pressure was $160 / 80$, heart rate was $92 \mathrm{bpm}$, respiratory rate was $18 \mathrm{x} / \mathrm{min}$, and $\mathrm{SaO} 2$ was $97 \%$. POCT Glucose was 90 and NIHSS score was 12. Code stroke was activated. Door to doctor was 5 minutes, door to CT was 10 minutes, door to decision was 20 minutes, 
and door to needle (IV-thrombolysis) was 58 minutes. Non-contrast CT scan showed large vessel occlusion in the right MCA with ASPECT score 5. IV thrombolysis was given using $0.6 \mathrm{mg} / \mathrm{kg}$ dose $(36 \mathrm{mg}$ ) within an hour. After thrombolysis, left extremities were able to move but still hemiparesis of the left extremities. Digital subtractional angiography (DSA) was performed and showed occlusion in M2 (TICI 1). Mechanical thrombectomy was performed using stent retriever. Three clots were successfully removed and achieve TICI 3. Patient was hospitalized in intensive care unit (ICU) for one day and stepped down to PACU. NIHSS post thrombectomy was 5 and $\mathrm{mRS}$ score at 2-week follow up was 3.
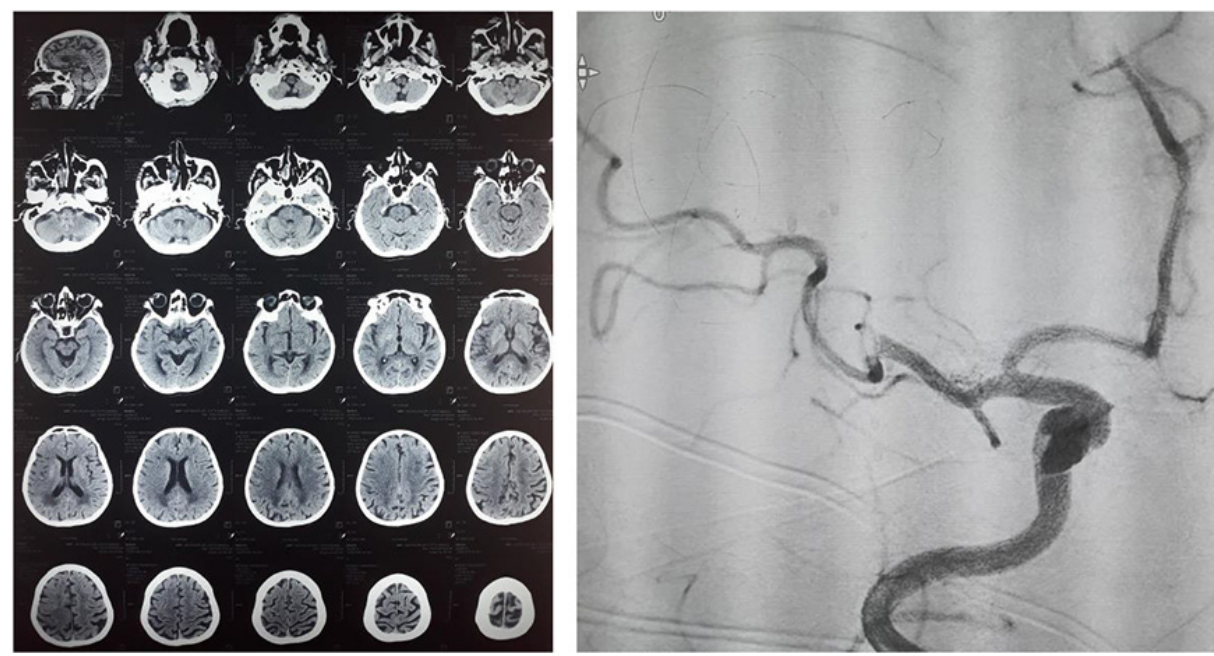

Figure 1. Non-contrast CT (left) and DSA before endovascular treatment showed TICI 1 in right MCA (right)

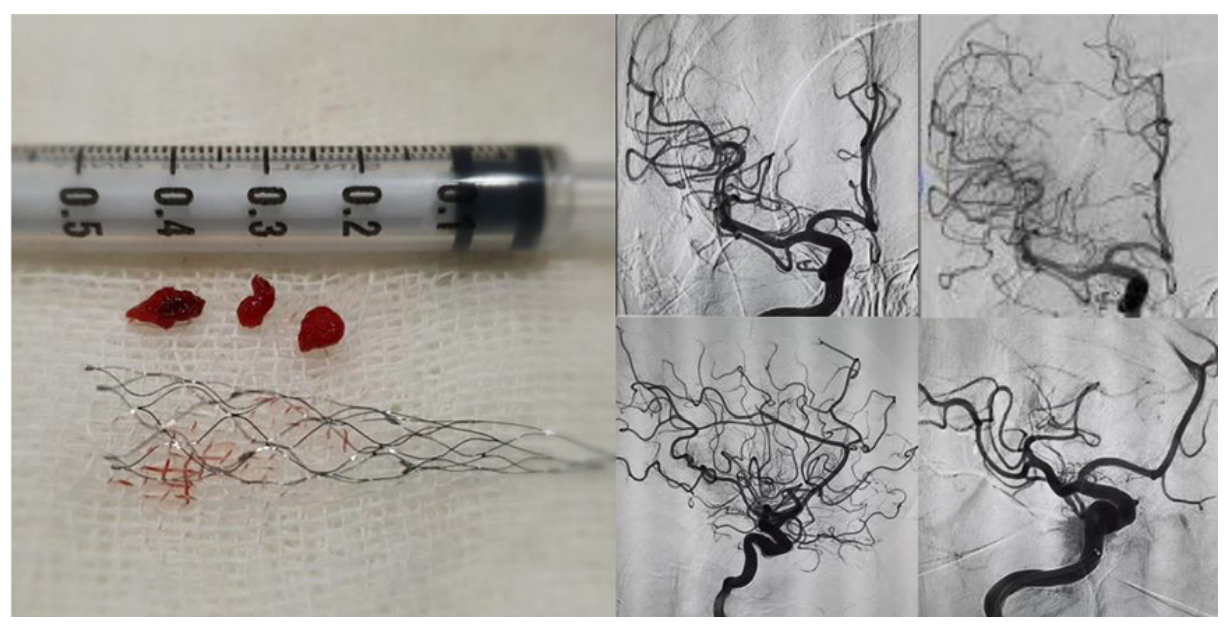

Figure 2. Clots removed through mechanical thrombectomy; stent retriever (left). TICI 3 was achieved after mechanical thrombectomy (right). reperfusion achieved in 56.7-78\%..$^{5-7}$ Complications such as $\mathrm{ICH}$ and other procedure-related complications count for $24 \%$ and $11 \%$ of all cases. Loneg-term follow up show poorer outcome in older patients, recovery of independent function can be expected in approximately 1 out of 4 cases. $^{6}$

The most powerful predictors of a good outcome across age groups are lower NIHSS at baseline, shorter delay between onset and reperfusion, and high ASPECT score on initial brain imaging. Achieving complete recanalization with a single thrombectomy device pass (firstpass effect) is a strong determinant of favorable outcome. ${ }^{7}$ However, there is no univariable analysis show significant result for predicting good functional outcome $(\mathrm{mRS}<2)$ at 90 days after procedure. ${ }^{8} \mathrm{~A}$ study including 124 nonagenarian patients showed that successful reperfusion after the first pass were associated with decrease in 90-day mortality and increase good outcome compared with patients without reperfusion. ${ }^{7}$ Complications occurred were embolic events, vessel perforations, vasospasm, dissections, groin hematomas, and subarachnoid hemorrhage. Intracranial hemorrhage within 24 hours occurred in $24-28 \%$ patients but only $4-8 \%$ considered as symptomatic, and there was no difference rates between patients with and without successful reperfusion. ${ }^{6,7,9}$

When compared with thrombolysis alone, nonagenarian patients who got endovascular treatment have lower NIHSS score at discharge, better mRS score at 90 days after procedure, and less symptomatic ICH. Although advanced age was reported to be associated with an increased tortuosity of the cerebral vasculature that increase technical difficulty of mechanical thrombecomy and resulting less complete recanalization, the use of latest generation of device can result in less complication. ${ }^{9}$ The first-line mechanical thrombectomy device strategy either stent retriever, contact aspiration, or either combination of both did not give significance in achieving successful reperfusion. ${ }^{7}$

\section{CONCLUSIONS}

Endovascular treatment with mechanical thrombectomy for large vessel occlusion in acute ischemic stroke can be performed 
in nonagenarian patient and have a high chance to achieve good reperfusion in most of the patients. Several predictor factors for having good outcome should be considered. However, several complications can still happen, especially

intracranial hemorrhage, but only a few showing symptoms.

\section{DECLARATIONS}

\section{Funding}

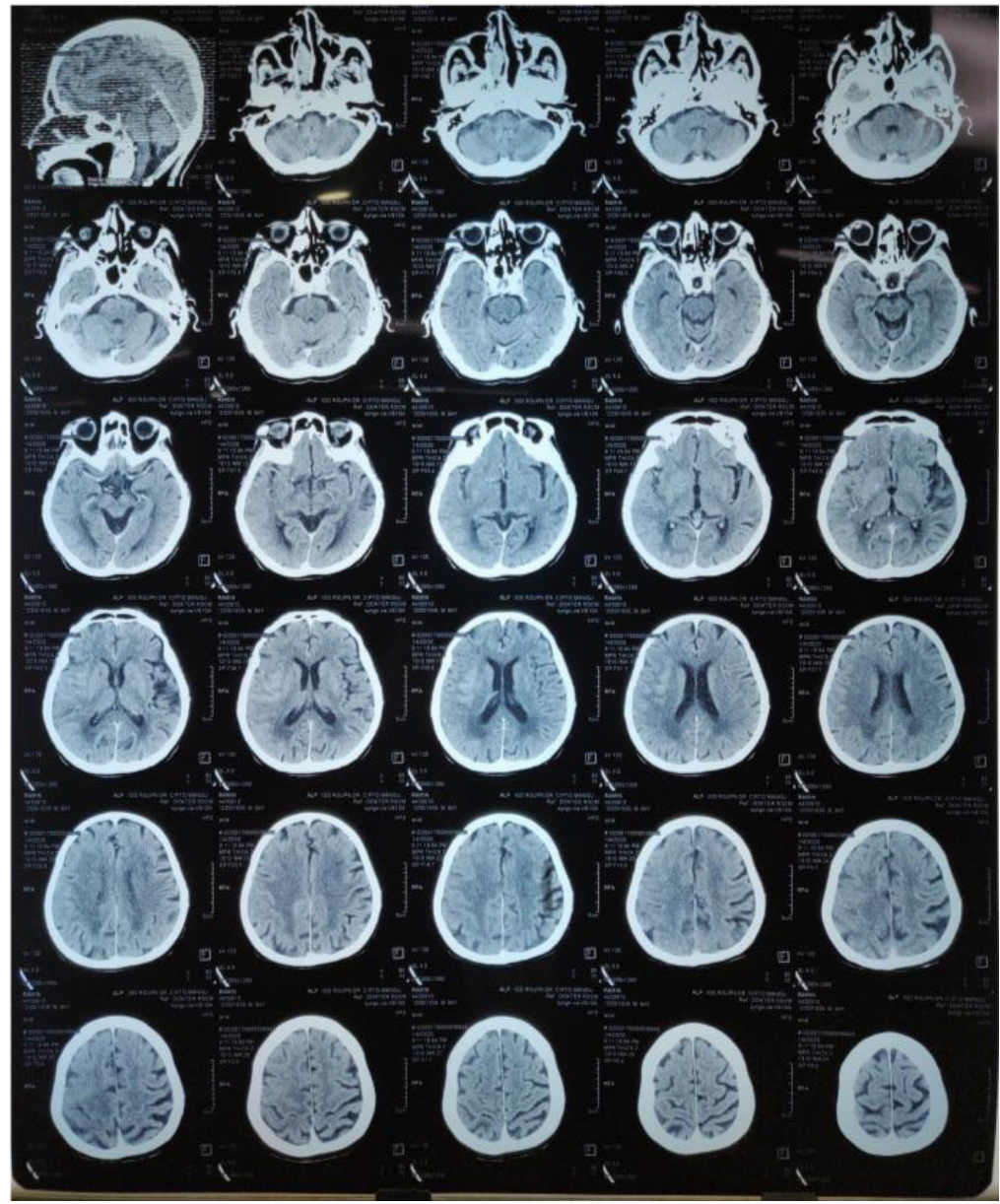

Figure 3. Post thrombectomy non-contrast CT scan showed bleeding transformation from ischemic lesion (right)

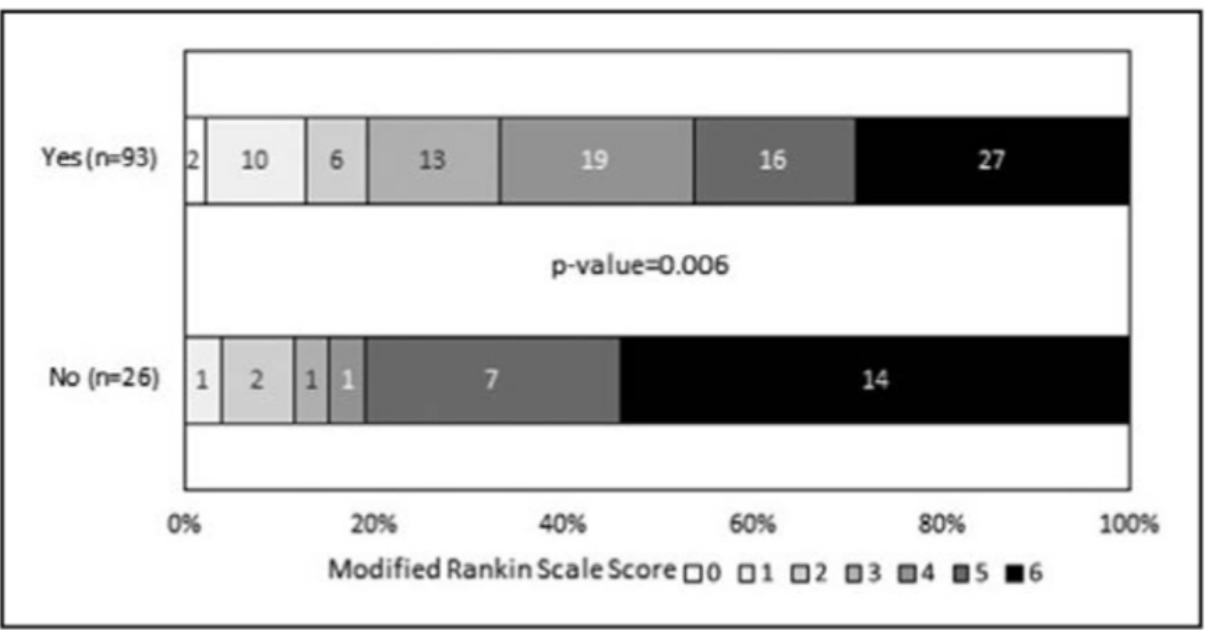

Picture 4. Distribution of $\mathrm{mRS}$ score at 90 days according to successful reperfusion status(7)

\section{Conflict of interest}

\section{Ethical approval}

\section{Authors' contributions}

Affan Priyambodo Permana, MD: consultant for the case and the operator during thrombectomy.

Zharifah Fauziyyah Nafisah, MD: second author write the article under supervision from first author

\section{REFERENCES}

1. Asia S. Indonesia: WHO statistical profile Indonesia : WHO statistical profile Distribution of causes of deaths DTP3 immunization among. 2015;15:2013-5.

2. Venketasubramanian N, Yoon BW, Pandian J, Navarro JC. Stroke Epidemiology in South, East, and South-East Asia: A Review. J Stroke. 2018;20(1):142-142.

3. Ackerson $\mathrm{T}$, Adeoye OM, Brown $\mathrm{M}$, Demaerschalk BM, Hoh B, Leslie-mazwi TM, et al. AHA / ASA Guideline 2018 Guidelines for the Early Management of Patients With Acute Ischemic Stroke. 2018. 46-99 p.

4. Alawieh A, Starke RM, Chatterjee AR, Turk A, Leacy R De, Rai AT, et al. Outcomes of endovascular thrombectomy in the elderly: a ' real-world ' multicenter study. 2019;(January 2013):545-53

5. Nagel XS, Bo XJ, Herweh XC, Ringleb XP, Bendszus XM, Stampfl XS. Endovascular Stroke Treatment of Nonagenarians. 2017;

6. Hilditch XCA, Nicholson XP, Murad XMH, Rabinstein XA, Schaafsma XJ, Pikula XA, et al. Endovascular Management of Acute Stroke in the Elderly: A Systematic Review and MetaAnalysis. 2018;1-5.

7. Rousiers ED, Lucas L, Richard S, Consoli A, Mazighi M, Labreuche J, et al. Impact of Reperfusion for Nonagenarians Treated by Mechanical Thrombectomy Insights From the ETIS Registry. 2019;1-6.

8. Meyer L, Alexandrou $M$, Leischner $\mathrm{H}$, Flottmann F, Deb-chatterji M, Abdullayev N, et al. Mechanical thrombectomy in nonagenarians with acute ischemic stroke. 2019;1-5.

9. Wu Q, Li Q, Huang C, Li Y, Wolff V, Qin $\mathrm{X}$. Efficacy and Safety of Endovascular Thrombectomy for Ischemic Stroke in Nonagenarians. 2019;1-8.

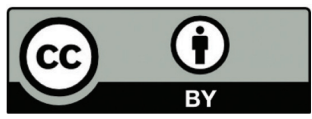

This work is licensed under a Creative Commons Attribution 